\title{
Bohemian Rhapsody: efeitos da atribuição de prémios cinematográficos no envolvimento no Facebook
}

\author{
Sónia de Almeida Ferreira \\ Escola Superior de Educação, Centro de Estudos em Educação, Tecnologias e Saúde, \\ Instituto Politécnico de Viseu, Portugal \\ Sara Santos \\ Escola Superior de Educação, Instituto Politécnico de Viseu, Portugal \\ Pedro Manuel do Espírito Santo \\ Escola Superior de Tecnologia e Gestão de Oliveira do Hospital, \\ Instituto Politécnico de Coimbra, Portugal
}

\begin{abstract}
Nowadays, the importance of social media in the various sectors of our society is unquestionable. Furthermore, the investment that the cinematographic industry has been making in the use of new digital platforms that promote communication with the public is also undeniable. The trend is the cinema converge with the Internet, so it is imperative that the cinematographic industry creates an online content production system continuously and updated before, during and after the film's debut in theatres. This empirical study aims to highlight the effects of the awarding of the best film and best actor in the 76th Golden Globe Award for the movie Bohemian Rhapsody. In this way, it was analysed the engagement of web users with the Facebook page before and after the attribution of the prizes to the film object of this analysis. The results show that as we move further from the release date of the movie, there will be a trend towards more "likes", but less "shares" and "comments" by users that follows the movie page. Nevertheless, this trend changed in order to the Bohemian Rhapsody victory in the Golden Globes. Therefore, it is concluded that the awarding of premiums to the films modifies the engagement of the users in that pages of social media.
\end{abstract}

Keywords: Film, Facebook, Social Media, Engagement

\section{Introdução}

As redes sociais mudaram o mundo e a forma como comunicamos. As marcas não ficaram indiferentes a essa mudança e estão presentes onde os seus consumidores estão: online. O mundo cinematográfico acompanhou esta evolução e os filmes são amplamente divulgados online (com grande enfoque nas redes sociais). O Facebook é a rede social mais utilizada em todo o mundo, com cerca de 2 mil milhões de utilizadores ativos (SproutSocial 2019), sendo que três quartos acede a esta rede diariamente. Por isso $97 \%$ das empresas que fazem anúncios utilizam esta plataforma. No consumo de vídeo, é a segunda plataforma preferida pelos utilizadores, logo a seguir ao Youtube (SproutSocial 2019). No entanto, o grande desafio para as marcas é criar envolvimento com os utilizadores, uma vez que este é em média de $0.09 \%$ (SproutSocial 2019). Devido ao algoritmo do Facebook que privilegia (entre vários fatores) o envolvimento dos utilizadores (gostos, comentários e partilhas), as marcas veem o seu alcance orgânico diminuir devido ao reduzido envolvimento (Brandwatch 2019).

Considerando este contexto, o estudo apresentado procura analisar os efeitos da estreia do filme Bohemian Rapsody e da atribuição dos prémios da $76^{a}$ edição dos Globos de Ouro no envolvimento dos seguidores na respetiva página de Facebook.

\section{Bohemian Rapsody: contextualização do filme}

O filme Bohemian Rapsody apresenta-se como uma celebração a Freddie Mercury, vocalista da banda britânica Queen, que "desafiou estereótipos [...] para se tornar um dos artistas mais amados" (Cinépolis 2018, 3).

Inicialmente a banda era composta por quatro elementos Brian May, Roger Taylor, John Deacon, interpretados por Gwilyn Lee, Ben Hardy, Joseph Mazzello, respetivamente, e por um vocalista que desistiu do conjunto - os Smile. Freddie Mercury, fã do grupo, tinha o sonho de ser vocalista profissional. Sabendo da desistência do vocalista, Mercury ofereceu-se para substituir o antigo vocalista. Aqui, e após algumas resistências dos restantes elementos à sua integração, nascem os Queen (Fuks, 2018).

O filme retrata, assim, o sucesso da banda através das "canções icónicas e som revolucionário", a sua quase implosão provocada pelo estilo de vida e Mercury e a apresentação triunfal no Live Aid - cena final do filme, onde o cantor, já padecendo de uma doença fatal, dirige a banda num momento épico da história do rock (Cinépolis 2018,4).

Com produção de Jim Beach, Graham King, Brian May, Peter Oberth, Bryan Singer, Roger Taylor e Robert De Niro e escrito por Anthony McCarten, o "roteiro contou com a supervisão de alguns dos membros da icônica banda e procurou ser o mais fiel possível aos fatos, embora muitas vezes o real tenha sido posto de lado em prol de uma versão mais ficcionalizada da história." (Fuks 2019, 90). Um tópico bastante criticado pelas fãs, foi a abordagem à sexualidade de Freddie. É mostrada, mas de forma bastante subtil, o que gerou descontentamento.

A realização do filme passou por alguma polémica e foi conduzida por três profissionais. Inicialmente a realização esteve entregue a Stephen Frears, demitido logo após a demissão de Sacha Baron Cohen, que iria interpretar o protagonista. O segundo realizador foi 
Bryan Singer, que também viria a ser despedido por desentendimentos com a equipa. Dexter Fletcher foi escolhido para o substituir e acabou por finalizar as filmagens e a pós-produção do filme.

A definição do protagonista também foi conturbada. Cohen, comediante de profissão, foi escolhido pela "semelhança física e talento e participou ativamente na criação do roteiro", até abandonar a equipa em 2013. A razão evocada foi a "clássica diferença criativa entre o ator e os membros do grupo" (Ramos 2017, 13). Depois de alguns anos suspenso, o projeto viria a contratar Be Whishaw, tendo sido demitido logo depois, sem que se saiba a razão. Em novembro de 2016 Rami Malek é confirmado como protagonista.

O desempenho de Malek no papel de Freddy é considerado, de facto, admirável (Ellis 2019), desde a caracterização física à apropriação da linguagem corporal do cantor. Esta interpretação valeu-lhe o Óscar 2019 de melhor ator na 91 a edição Academy Awards 2019.

A este prémio, o filme soma na mesma cerimónia as estatuetas de Melhor Montagem/Edição - John Ottman, Melhor Edição de Som - Nina Harysyone e John Warhurst, e Melhor Mistura de Som - Paul Massey, Tim Cavagin e John Casali, estando também nomeado para melhor filme. Bohemian Rhapsody venceu também o Globo de Ouro na $76^{a}$ edição dos Globos de Ouro - momento em análise neste estudo para Melhor Filme Dramático e Melhor Ator em Filme Dramático e esteve indicado para melhor filme no Producers Guild of America e no BAFTA. Rami Malek também venceu os prémios de Melhor Ator no BAFTA e no Screen Actors Guild.

\section{Revisão de literatura}

Os social media influenciam diariamente as pessoas e, consequentemente, as marcas e organizações. Estas têm implicações na maioria dos aspetos da vida pessoal (seja em questões sociais, políticas, educacionais, etc) (Alalwan et al. 2016; Algharabat et al. 2017).

Kaplan e Haenlein (2010) identificaram seis tipos de plataformas de social media: blogs, projetos colaborativos (como a Wikipedia), comunidades de conteúdo (por exemplo, YouTube), mundos virtuais (por exemplo, World of Warcraft e Second Life) e redes sociais. Estas últimas são definidas por Boyd e Ellison (2007, 211) como "serviços baseados na web que permitem que os indivíduos (1) construam um perfil público ou semi-público dentro de um sistema limitado, (2) articulem uma lista de outros utilizadores com os quais partilham uma conexão e (3) visualizem e percorram a sua lista de conexões e as publicações aquelas feitas por outras pessoas dentro do sistema".

No entanto, é necessário analisar os social media nos seus diversos contextos (Hutchins 2014) e as marcas têm utilizado as suas ferramentas para gerar notoriedade, envolvimento e relevância (Hutter et al. 2013).

Na última década o envolvimento do consumidor tem sido estudado por vários autores (Hollebeek et al. 2014). O envolvimento nos social media é um contexto especifico de envolvimento do consumidor (Brodie et al. 2013) e que começa com o "gostar" de uma página no Facebook (Beukeboom et al. 2015).

O envolvimento nas redes sociais está dividido em 3 níveis: 'gostos', 'comentários' e 'partilhas' (Peters et al. 2012). Quando o utilizador faz "gosto" aparece no "feed" dos seus "amigos" pelo que pode ser considerado equivalente ao passa-palavra positivo (Swani et al. 2013).

Muntinga et al. (2011) distinguem o envolvimento do consumidor nas redes sociais em 3 tipos de comportamento: consumo (os consumidores leem informação, veem imagens e vídeos, comentários e criticas, num tipo de comportamento passivo lurker); contribuição quando os utilizadores gostam ou comentam fotografias e vídeos e se envolvem em conversas sobre a marca; criação - os utilizadores produzem e publicam conteúdos relacionados com a marca, preconizado no modelo Consumer Online Brand-Related Activity (COBRA). No nível de "criação" os utilizadores criam proactivamente conteúdos sobre as marcas nas suas redes pessoais (Men \& Tsai, 2014). No entanto, Azar et al. (2016) considera que no Facebook é difícil distinguir "contribuição" e "criação" de acordo com os estudos de Muntinga et al. (2011). Neste sentido, autores como Tsai e Men (2013) aplicam a tipologia de Muntinga et al. (2011) ao contexto de Facebook utilizando apenas as variáveis "consumo" e contribuição".

No comportamento de "consumo", o utilizador é passivo, apenas "observa", ou seja, tem o comportamento de "lurking", muito frequente nas redes sociais (Utz e Beukeboom 2011). Mas, como consequência esse comportamento levará à diminuição visibilidade orgânica da página nas redes sociais pelo que as marcas devem estimular os utilizadores a tornarem-se mais ativos (Sun et al. 2014).

O envolvimento nas redes sociais tem consequências relevantes para as marcas. Hudson et al. (2016) concluíram que os consumidores que se envolvem nas redes sociais com as suas marcas preferidas têm relações mais fortes com as mesmas. Na mesma perspetiva, Cummins et al. (2014) consideram que este envolvimento contribuí para aumentar a satisfação, lealdade e retenção dos clientes. Alealdade e a intenção de compra são, então, influenciadas pelo envolvimento nas redes sociais (Kim e Ko 2012). No entanto, criar envolvimento com as publicações nas redes sociais não é fácil para as marcas. Os consumidores tendem a envolverem-se mais com conteúdos não comerciais e que incluam questões emocionais e sentimentos (Swani et al. 2013).

\section{Motivações para participar nas redes sociais}

Estar presente nas redes sociais faz parte da estratégia de muitas marcas e organizações. Mas e os utilizadores, porquê participam e interagem nestas redes? Vários são os motivos apresentados que passam por:

- procura de informação - os utilizadores utilizam as redes sociais para procurar informação sobre marcas ou produtos (Brodie et al. 2013; Mangold e 
Faulds 2009). Muntinga et al. (2011) consideram que o comportamento passivo de "lurking" (observar) está relacionado com este motivo.

- entretenimento - quer para o comportamento lurking quer para o de ativo, o entretenimento (relaxar, alivio emocional, prazer cultural ou estético) é uma das principais motivações (Muntinga et al. 2011).

- recompensa - "O grau em que os membros da comunidade querem ganhar recompensas utilitárias (por exemplo, recompensas monetárias, economia de tempo, ofertas ou incentivos, merchandising e prémios) através da sua participação na comunidade» (Baldus et al. 2015, 981).

Tsai e Men (2013) consideram que os incentivos económicos são das primeiras razões para os utilizadores usarem as páginas de Facebook. No entanto, Muntinga et al. (2011) consideram que é um motivo para consumir conteúdo, mas não para contribuir ou criar conteúdo relacionado com a marca.

- integração e interação social - quando contribuem e criam conteúdos nas redes sociais os consumidores relacionam-se com os outros (Muntinga et al. 2011), sentindo-se parte da comunidade e importantes (Daugherty et al. 2008). Quando os utilizadores apenas consomem conteúdo não se verifica esta necessidade de interação social (Muntinga et al. 2011).

Segundo Schau e Gilly (2003) os consumidores utilizam as marcas para criar uma identidade própria, reforçar a sua auto-estima e ao expressarem-se no Facebook conseguem relacionar-se e criar uma impressão nos outros (Muntinga et al. 2011). Assim, a influência social (influenciar outros consumidores da marca) (Muntinga et al., 2011) e o criar laços (Azar et al. 2016) são os principais motivos para se envolverem nas redes sociais.

Por outro lado, Gummerus et al. (2012) dividem os benefícios das redes sociais em: sociais, funcionais e hedónicos. São estes benefícios que levam as pessoas a interagir em comunidades online.

No entanto, as motivações mudam ao longo do tempo (Shao e Ross 2015). Estes autores consideram que os primeiros motivos para os consumidores participarem nas comunidades são o socializar a adquirir informação e mais tarde o entretenimento. Posteriormente a informação sobre produtos e marcas volta a assumir importância (Shao e Ross 2015).

\section{Resultados}

Este estudo seguiu uma metodologia que se baseou na análise de publicações na rede social Facebook em duas fases.

Numa primeira fase, estudou-se os efeitos da estreia do filme no envolvimento dos seguidores no Facebook e, numa segunda fase, os impactos da atribuição de prémios de melhor filme e melhor ator na $76^{a}$ edição dos Globos de Ouro ao filme Bohemian Rhapsody.

\section{Estudo 1}

No primeiro estudo iniciou-se a análise no dia 19 de outubro de 2019 e terminou-se a 11 de dezembro de 2019. Durante este período obtivesse informação de 55 publicações. Para ser possível analisar algumas diferenças de comportamento nas redes sociais, em específico na página de Facebook do filme Bohemian Rhapsody, dividiu-se as publicações em 2 grupos: antes da data de estreia do filme (3 de novembro de 2019) e após essa data de estreia. As publicações que os gestores da página de Facebook do filme Bohemian Rhapsody fizeram antes da sua estreia foram 14 vídeos sobre partes do filme ou entrevistas a atores, 3 imagens e 6 partilha de links externos.

Após a estreia do filme foram analisados 14 vídeos, 7 imagens e 1 partilha de link externo.

A Tabela 1 resume a informação recolhida. Desta tabela é possível analisar que o número médio de gostos é significativamente maior após a estreia do filme.

\begin{tabular}{|l|l|l|}
\hline & $\begin{array}{l}\text { Mês seguinte } \\
\text { após } \\
\text { lançamento } \\
\text { filme }\end{array}$ & $\begin{array}{l}\text { Mês } \\
\text { anterior ao } \\
\text { Lançamento } \\
\text { Filme }\end{array}$ \\
\hline n Publicações & 22 & 23 \\
\hline Gostos & 99100 & 85605 \\
\hline Comentários & 5005 & 7838 \\
\hline Partilhas & 20971 & 46615 \\
\hline Média de gostos & 4504,55 & 3721,96 \\
\hline $\begin{array}{l}\text { Média de } \\
\text { comentários }\end{array}$ & 227,50 & 340,78 \\
\hline Média de partilhas & 953,23 & 2026,74 \\
\hline
\end{tabular}

Tabela 1 - análise do envolvimento no Facebook antes e depois da estreia do filme

Contudo o envolvimento nos comentários vinculados no Facebook é menor após a estreia do filme, além do número de partilhas. Assim, os efeitos de estreia do filme Bohemian Rhapsody são evidentes no envolvimento dos seguidores no Facebook, pela diferença significativa no que diz respeito à atividade online. Verifica-se um maior envolvimento nos comentários e partilhas em datas anteriores à estreia do filme, pela curiosidade que o filme permite despertar nos seus públicos.

\section{Estudo 2}

A segunda parte do estudo procura evidenciar os efeitos da atribuição de prémios ao filme Bohemian Rhapsody no envolvimento no Facebook.

Para este segundo estudo, optou-se por analisar as publicações que foram divulgadas na página do Facebook do filme entre 1 de janeiro de 2019 e 12 de janeiro de 2019.

A cerimónia dos Globos de Ouro decorreu em 7 de janeiro de 2019 e a atribuição do prémio de melhor filme e melhor ator provocou um maior envolvimento dos seguidores da página.

Das 6 publicações feitas na página de Facebook do filme, 4 são vídeos, 1 sondagem e 1 imagem.

Nos 6 dias seguintes à cerimónia dos globos de 
Ouro, o filme publicou 11 vezes, das quais 5 vezes postou vídeos, 5 vezes divulgou imagens e por 1 vez partilhou um link externo.

Na tabela 2 estão os dados apresentados, separados entre as publicações anteriores à cerimónia dos globos de ouro e as publicações após este momento.

\begin{tabular}{|l|l|l|}
\hline $\begin{array}{l}6 \text { dias antes } \\
\text { anúncio } \\
\text { vencedores } \\
\text { Globos }\end{array}$ & $\begin{array}{l}6 \text { dias após } \\
\text { anúncio de } \\
\text { vencedores } \\
\text { Globos }\end{array}$ \\
\hline $\mathrm{n}^{\circ}$ Publicações & 6 & 11 \\
\hline Gostos & 11003 & 35672 \\
\hline Comentários & 549 & 1359 \\
\hline Partilhas & 1877 & 3441 \\
\hline Média de gostos & 1833,83 & 3242,91 \\
\hline Média de & 91,50 & 123,55 \\
\hline comentários & & 312,82 \\
\hline Média de partilhas & 312,83 & \\
\hline
\end{tabular}

Tabela 2 - análise do envolvimento no Facebook antes e depois da atribuição do prémio de melhor filme e melhor ator na $76^{a}$ edição dos Globos de Ouro

Apesar de a página do Facebook do filme ter maior atividade pelo número de publicações partilhadas, os seguidores reagiram de uma forma mais entusiasta às partilhas. Verifica-se pelos resultados da tabela 2 que o número médio de gostos por publicação quase que dobrou e a média de comentários por publicação é significativamente maior.

Apesar de o número médio de partilhas por publicação ser praticamente constante e não variar com a atribuição de prémios nos globos de Ouro, o envolvimento é maior após o filme receber prémios reconhecidos internacionalmente como é o caso dos globos de Ouro.

A comparação dos 2 estudos permite verificar que o número médio de gostos aumenta para níveis próximos daqueles que foram antes da estreia do filme, contudo, os comentários apesar de aumentarem ficam longe do número médio de comentários que aconteceu no período antes da estreia do filme.

\section{Conclusão}

O lançamento de novos filmes são momentos marcantes para os amantes da atividade cinematográfica. Nos últimos anos, associado ao lançamento de novos filmes, os produtores habitualmente lançam nas redes sociais publicações que procuram despertar a curiosidade dos seguidores do filme e procuram atrair os públicos que ainda não seguem o filme a ser lançado.

Neste sentido, o estudo aqui apresentado foi desenvolvido em duas partes e procurou analisar os efeitos da estreia e os efeitos da atribuição de prémios ao filme Bohemian Rhapsody na atividade no Facebook.
Dos resultados, encontrou-se diferença de comportamento dos seguidores antes e depois dos momentos relatados.

Assim, as conclusões principais deste trabalho, retratam que o envolvimento dos seguidores do Facebook é maior nos momentos anteriores à estreia do filme. Este maior envolvimento reflete-se no número de comentários existentes. O número de comentários dos seguidores do filme no Facebook é maior antes da estreia do filme e este número de comentários vai decrescendo após a estreia do filme. Esta tendência apenas é modificada quando o filme recebe algum prémio relevante como é o caso dos globos de ouro.

No caso de o filme ser premiado, a interação através do número de comentários aumenta, modificando a tendência decrescente até então verificada.

$\mathrm{Na}$ análise ao número de partilhas, concluímos que a tendência é semelhante ao verificado no envolvimento através de comentários. Neste caso, os seguidores do filme após a estreia do filme partilham menos à medida que nos afastamos da data de estreia. Neste caso em concreto, não é relevante a atribuição de prémios ao filme, mesmo que se trate dos prémios de elevada notoriedade como é o caso dos Globos de Ouro. Concluímos que a atribuição de prémios não influencia o envolvimento dos seguidores por via das partilhas efetuadas.

A terceira conclusão que retirámos do envolvimento à página do Facebook do filme Bohemian Rhapsody está relacionada com o número de "Likes". Neste âmbito, o nosso estudo evidencia que a estreia do filme aumenta o número médio de "likes" às publicações do filme que após algum tempo decorrido esse número decresce. Contudo, a atribuição de prémios como os Globos de Ouro provoca um maior envolvimento dos internautas no Facebook à página do filme.

Em resumo, o nosso estudo conclui que a atribuição de prémios aos filmes, em particular os globos de ouro, promove modificações no envolvimento à página de Facebook do filme.

\section{Agradecimentos}

Agradecimento ao Instituto Politécnico de Viseu, Centro de Estudos em Educação, Tecnologias e Saúde (CI\&DETS) e à Fundação para a Ciência e a Tecnologia (FCT).

\section{Referências bibliográficas}

Alalwan Ali Abdallah, Rana Nriprenda, Algharabat Raed, Tarhini Ali. 2016. A Systematic Review of Extant Literature in Social Media in the Marketing Perspective. In: Dwivedi Y. et al. (eds) Social Media: The Good, the Bad, and the Ugly. I3E 2016. Lecture Notes in Computer Science, 9844. Springer, Cham.

Algharabat Raed, Alalwan Abdadallah Ali, Rana P. Nriprenda, Dwivedi, K. Yogesh. 2017. "Three dimensional product presentation quality antecedents and their consequences for online retailers: The moderating role of virtual product experience" in Journal of Retailing and Consumer Service, 36, 203-217.

Azar L. Salim, Machado Joana César, Vacas-de- 
Carvalho Leonor, Mendes Ana. 2016. "Motivations to interact with brands on Facebook-Towards a typology of consumer-brand interactions" in Journal of Brand Management, 23, 153-178.

Baldus J. Brian, Voorhees Clay, Calantone Roger. 2015. "Online brand community engagement: Scale development and validation" in Journal of Business Research 68, n5: 978-985.

Beukeboom J. Camiel, Kerkhof Peter, de Vries Metter. 2015. "Does a virtual like cause actual liking? How following a brand's Facebook updates enhances brand evaluations and purchase intention" in Journal of Interactive Marketing, 32, 26-36

Boyd M. Danah, Ellison B. Nicole. 2007. "Social network sites: Definition, history, and scholarship" in Journal of Computer Mediated Communication 13, n¹: 210-230.

Brandwatch. 2019. Tha Facebook algorithm explained. https://www.brandwatch.com/blog/the-facebook-algorithmexplained. Acedido em 14 de maio de 2019.

Brodie J. Roderick, Ilic Ana, Juric Biljana, Hollebeek Linda. 2013. "Consumer engagement in a virtual brand community: An exploratory analysis" in Journal of Business Research, 66, 105-114.

Cinépolis. 2018. Bohemian Rhapsody. https://www. cinepolis.com.br/filme/9823-bohemian-rhapsody.html. Acedido em 10 de maio de 2019.

Cummins Shannon, Peltier W. James, Schibrowsky A. John, Nill Aelxander. 2014. "Consumer behavior in the online context" in Journal of Research in Interactive Marketing 8, n³: 169-202.

Daugherty Terry, Eastin S. Matthew, Bright Laura. 2008. "Exploring consumer motivations for creating usergenerated content" in Journal of Interactive Advertising 8, n²: 1-24.

Ellis, Nick. 2019. Resenha - Bohemian Rhapsody exagera na ficção, mas é belo tributo a Freddie Mercury. https://meiobit.com/396012/resenha-bohemian-rhapsodyfreddie-mercury-queen/. Acedido em 10 de maio de 2019.

Fuks, Rebeca. 2019. Filme Bohemian Rhapsody. https://www.culturagenial.com/filme-bohemian-rhapsody/. Acedido em 10 de maio de 2019.

Gummerus Johanna, Liljander Veronica, Weman Emil, Pihlström Minna. 2012. "Customer engagement in a Facebook brand community" in Management Resources Review 35, n9: 857-877.

Kaplan M. Andreas, Haenlein Michael. 2010. "Users of the world, unite! The challenges and opportunities of Social Media" in Business Horizons 53, n¹: 59-68

Kim J. Angella, Ko Eunju. 2012. "Do social media marketing activities enhance customer equity? An empirical study of luxury fashion brand" in Journal of Business Research 65, n¹0: 1480-1486

Hollebeek D. Linda, Glynn S. Mark, Brodie J. Roderick. 2014. "Consumer brand engagement in social media: conceptualization, scale development and validation" in Journal of Interactive Marketing 28, n²: 149-165.

Hudson Simon, Huang Li, Roth S. Martin, Madden J. Thomas. 2016. "The influence of social media interactions on consumer-brand relationships: a three-country study of brand perceptions and marketing behaviors" in International Journal of Research in Marketing 33, $n^{\circ} 1$ : 27-41.

Hutchins, Brett. 2014. "Twitter: follow the Money and look beyond sports" in Communication \&Sports, 2, 122-126

Hutter Katja, Hautz Julia, Dennhardt Severin, Füller Johann. 2013. "The impact of user interactions in social media on brand awareness and purchase intention: The case of MINI on Facebook" in Journal of Product \& Brand
Management 22, n5/6: 342-351

Mangold W. Glynn, Faulds J. David. 2009. "Social Media: The New Hybrid Element of the Promotion Mix" in Business Horizons 52, $n^{\circ}$ 4: 357-365

Men R. Linjuan, Tsai Wanhsiu Sunny. 2014. Perceptual, attitudinal, and behavioral outcomes of organizationpublic engagement on corporate social networking sites in Journal of Public Relations Research 26, 417-435.

Muntinga Daniël, Moorman Marjolein, Smit Edith. 2011. "Introducing COBRAs: Exploring motivations for brand-related social media use" in International Journal of Advertising, 30, $\mathrm{n}^{\circ}$ 1: 13-46.

Peters Kay, Chen Yubo, Kaplan M. Aandreas, Ognibeni Björn, Pauwels Koen. 2012. "Social media metrics - A framework and guidelines for managing social media" in Journal of Interactive Marketing 27, n4: 281-298

Ramos, Luciana. 2017. A demissão de Bryan Singer é apenas mais um problema na produção de Bohemian Rhapsody. https://maisquecinema.com.br/demissao-debryan-singer-e-apenas-mais-um-problema-na-producaode-bohemian-rhapsody/. Acedido em 10 de maio de 2019.

Schau Hope Jansen, Gilly Mary. 2003. "We are what we post? Self-presentation in a personal web space" in Journal of Consumer Research 30, n 3: 385-404.

Shao Wei, Ross Mitchell. 2015. "Testing a conceptual model of Facebook brand page communities" in Journal of Research in Interactive Marketing 9, n³: 239-258.

SproutSocial. 2019. 65 social media statistics to bookmark in 2019. https://sproutsocial.com/insights/socialmedia-statistics/. Acedido em 14 de maio de 2019.

Sun Na, Rau Patrick, Pei-Luen Ma Liang. 2014. "Understanding lurkers in online communities: A literature review" in Computers in Human Behavior, 38, 110-117.

Swani Kunal, Milne George, Brown P. Brian. 2013. "Spreading the word through likes on Facebook" in Journal of Research in Interactive Marketing 7, $n^{\circ} 4$ : 269-294

Tsai Wanhsiu Sunny, Men Linjuan Rita. 2013. "Motivations and Antecedents of Consumer Engagement With Brand Pages on Social Networking Sites" in Journal of Interactive Advertising 13, $\mathrm{n}^{\circ} 2$ : 76-87.

Utz Sonja, Beukeboom J. Camiel. 2011. "The role of social network sites in romantic relationships: Effects on jealousy and relationship happiness" in Journal of Computer Mediated Communication 16, $n^{\circ} 4$ : 511-527. 